\title{
ciência
lurạl
}

\section{PERCEPÇÕES DE GESTANTES SOBRE A PROMOÇÃO DO PARTO NORMAL NO PRÉ-NATAL}

\section{Perception of pregnant women about the promotion of normal birth in the prenatal}

Cintia Danielle Faustino da Silva Guedes - Enfermeira Especialista em Gestão Hospitalar (CENPEX). E-mail: cintiaguedesenfa@gmail.com

Thuanne Karla Carvalho de Souza - Enfermeira da ESF Antônio Joaquim (SMS - Vila Flor/RN). E-mail: thuanne.souzaa@gmail.com

Luan Nogueira Bezerra de Medeiros - Enfermeiro e Pós-graduando no Programa de Residência Multiprofissional em Assistência Materno-Infantil (UFRN). E-mail: luan-nogueira91@hotmail.com

Deyvisson Ribeiro da Silva • Enfermeiro e Pós-graduando em Urgência e Emergência (ESTÁCIO DE SÁ) e docente (UNP).E-mail: deyvisson_drs@hotmail.com

Belisana Pinto de Abreu Araújo Neta - Enfermeira e Docente (ESTÁCIO DE SÁ). E-mail: b.belisana@gmail.com

Maria Margarida dos Santos - Enfermeira e Pós-graduanda em Terapia Intensiva Neonatal (CENPEX). E-mail: m_margaridasantos@hotmail.com

Alex-sandra Barbosa da Costa • Enfermeira e Pós-graduanda no Programa de Residência em Saúde da Criança (IMIP).E-mail: alex-sandra-jesus@hotmail.com

Autora responsável pela correspondência:

Cintia Danielle Faustino da Silva Guedes. E-mail: cintiaguedesenfa@gmail.com 
Introdução: O parto normal deve ser estimulado na assistência pré-natal. Objetivo: Conhecer a percepção de gestantes, de uma equipe da Estratégia de Saúde da Família de Parnamirim/RN, sobre a promoção do parto normal no pré-natal. Métodos: Trata-se de um estudo exploratório, descritivo e de abordagem qualitativa. Foram realizadas entrevistas com 17 gestantes e analisadas pela análise de conteúdo. Resultados: As gestantes relataram que existem poucas atividades educativas de promoção ao parto no pré-natal; apontaram a necessidade de melhor comunicação por parte dos profissionais; demonstraram entender a importância da preparação na gravidez para o parto, mesmo as orientações e informações sendo falhas; e contribuíram com opiniões de métodos educativos para promoção do parto normal no pré-natal. Conclusões: Recomenda-se a realização de ações educativas em grupo e estratégias para o fortalecimento de vínculo entre a equipe e as gestantes, buscando uma melhor promoção ao parto normal e assistência pré-natal de qualidade.

Palavras-chave: Promoção da Saúde; Cuidado Pré-Natal; Parto Normal.

\section{ABSTRACT}

Introduction: Normal birth should be stimulated in prenatal care. Objective: To know the perception of pregnant women, from a team of the Family Health Strategy of Parnamirim/RN, on the promotion of normal prenatal delivery. Methods: This is an exploratory, descriptive and qualitative approach. Interviews were conducted with 17 pregnant women and analyzed by content analysis. Results: Pregnant women reported that there are few educational activities to promote prenatal delivery; pointed out the need for better communication by professionals; have demonstrated the importance of preparing pregnancy for childbirth, even when guidelines and information are flawed; and contributed with opinions of educational methods to promote normal prenatal delivery. Conclusions: It is recommended to carry out group educational actions and strategies to strengthen the bond between the team and the pregnant women, seeking a better promotion of normal delivery and quality prenatal care.

Key words: Health Promotion; Prenatal Care; Natural Childbirth 


\section{Introdução}

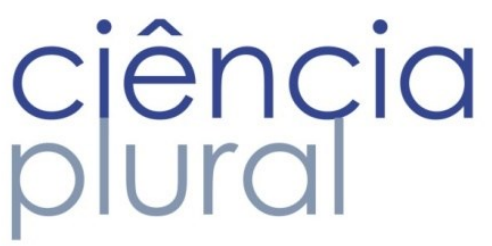

Atualmente a assistência obstétrica emergiu para o centro dos debates sobre saúde pública no Brasil, fomentado pelos mais diversos setores da sociedade, fortificado e embasado pelo sentimento de busca da humanização e do resgate do parto normal11-2.

No Brasil cerca de $52 \%$ do total de partos são cesarianas, dados que figuram o país com maior índice de cesáreas do mundo, contrapondo-se à recomendação da Organização Mundial da Saúde de que somente 10 a 15\% dos partos sejam realizados por via cirúrgica ${ }^{3-4}$. Existe um grande número de cesáreas mesmo em parturientes com gestação de risco habitual, o que evidencia a realização desse procedimento sem que existam verdadeiras indicações e, aproximadamente, $80 \%$ desses partos são realizados sem que a gestante tivesse entrado em trabalho de parto ${ }^{1,3}$.

Vários são os fatores que podem influenciar para o parto cesáreo, mas é preciso que os profissionais de saúde estejam capacitados e qualificados para acompanhar a mulher durante o pré-natal, garantindo boas práticas na promoção do parto norma|5-6.

Pode-se destacar o Programa de Humanização do Pré-Natal e Nascimento (PHPN), criado em 2000, que busca melhoria na qualidade do atendimento pré-natal, resgatando o papel central e ativo da mulher no parto ${ }^{7}$. Neste sentido torna-se imprescindível citar a Rede Cegonha, que é um modelo de atenção à saúde materno-infantil caracterizada pelo acompanhamento da mulher desde o planejamento reprodutivo, gravidez, parto, nascimento e puerpério ${ }^{8}$.

Considerando as características preconizadas para a assistência no pré-natal, percebe-se que 0 cuidado à gestação configura um dos principais indicadores do prognóstico do nascimento, importância justificada pelo seu caráter investigativo de agravos e pelo seu destacável papel de promoção à saúde ${ }^{9}$. No entanto, existem falhas durante a assistência no pré-natal relacionadas às informações que as mulheres devem receber sobre as boas práticas e cuidados obstétricos adequados, sobre os benefícios do parto vaginal, e serem orientadas e preparadas para conduzirem o seu parto1.

Partindo desta premissa e considerando que a mudança do modelo de assistência ao parto no Brasil abarca vieses que precisam ser entendidos e contemplados pelas políticas públicas de saúde e pelos profissionais envolvidos nesse processo, torna-se imprescindível evidenciar a visão da mulher gestante sobre os serviços à ela direcionados, como forma de propor adequações concernentes ao fortalecimento das orientações e incentivo ao parto normal no pré-natal, estimulando a escolha da gestante pelo parto fisiológico. Diante do exposto, formulou-se a seguinte pergunta norteadora: qual a percepção de gestantes sobre a promoção do parto normal no pré-natal? Assim, a presente pesquisa se propôs a conhecer a 


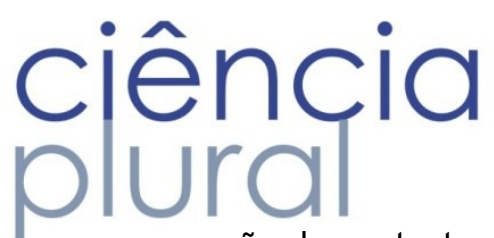

percepção de gestantes, de uma equipe da Estratégia de Saúde da Família de Parnamirim/RN, sobre a promoção do parto normal no pré-natal.

\section{Metodologia}

Trata-se de um estudo exploratório e descritivo, com abordagem qualitativa. A pesquisa foi realizada em uma equipe da Estratégia de Saúde da Família (ESF) de uma Unidade Básica de Saúde (UBS) do município de Parnamirim, Estado do Rio grande do Norte, Brasil, que atende um número de aproximadamente 1608 famílias adscritas. A população do estudo foi de gestantes cadastradas para consultas de pré-natal na ESF durante o mês de outubro de 2015. Neste mês, 32 gestantes realizavam consultas de pré-natal.

Os critérios de inclusão para participação na pesquisa foram: gestantes com idade maior de 18 anos e que estivessem entre o segundo e terceiro trimestre do período gestacional. A escolha desse último critério justifica-se pelo fato de que neste período a gestante já tenha participado de consultas e atividades do prénatal. Os critérios de exclusão foram: gestantes menores de 18 anos de idade e que estivessem no primeiro trimestre de gestação. Sob estes aspectos, duas gestantes não se enquadraram na pesquisa por serem menores de 18 anos de idade. Houve perda de 13 gestantes por recusa à participação. Assim, totalizou um número de 17 sujeitos participantes.

Utilizou-se um instrumento semiestruturado elaborado com questões fechadas e abertas para realização da entrevista. Esse era constituído por informações socioeconômicas das gestantes e abordava também aspectos sobre a preferência do tipo de parto e a percepção sobre a promoção do parto normal no pré-natal. A coleta dos dados ocorreu antes ou após as consultas de pré-natal, de forma individualizada em um espaço da UBS que promoveu privacidade e conforto às participantes.

Os dados foram analisados na perspectiva da análise de conteúdo, cujo foco é investigar um tema que se pretende entender, explorando um conjunto de opiniões e representações sociais. Esse método de análise abrange cronologicamente três fases: Pré-análise; Exploração do Material; e Tratamento dos Resultados e Interpretação ${ }^{10}$.

Seguindo na análise, na primeira fase, procederam-se leituras minuciosas através das quais foram definidas duas categorias temáticas como unidades de registro, além dos trechos mais significativos. As primeiras leituras possibilitaram uma impressão dos resultados das entrevistas. 
$\mathrm{Na}$ segunda fase, as falas foram codificadas, procurando-se compreender seus significados nas frases e expressões faladas, de forma que foi possível classificá-las e reuni-las em grupos de sentidos, denominados categorias. A categorização por meio de uma tabela possibilitou o melhor entendimento das percepções que emergiam nas falas das gestantes, além do processo de contagem de frequência. Neste processo, as unidades com maior frequência de aparição ganharam importância e foram agrupadas para a formação de subcategorias.

$\mathrm{Na}$ terceira etapa realizou-se a análise e interpretação do material, inferindo-se significados. Os resultados seguem apresentados e simultaneamente discutidos, embasando-se na literatura científica pertinente.

Para garantir o anonimato das participantes adotou-se a identificação por pseudônimos de flores, em alusão à beleza do processo de transformação vivenciado pelas mulheres através do processo gravídico. Este estudo atendeu a resolução 466/2012 e foi aprovado pelo Comitê de Ética e Pesquisa da Universidade Federal do Rio Grande do Norte, da cidade de Natal - RN, através do parecer No 1.257.931.

\section{Resultados}

Através do processo de exploração do material, na técnica de análise temática utilizada no conteúdo das entrevistas, emergiram duas categorias temáticas e subcategorias, mas apenas a categoria "Promoção do Parto Normal no Pré-Natal" foi utilizada para elaboração deste artigo. Nesta categoria serão discutidos os aspectos relacionados às percepções das entrevistadas quanto ao pré-natal e atividades desenvolvidas de incentivo e preparo da gestante para o parto normal. A figura 1 ilustra o plano de análise. 


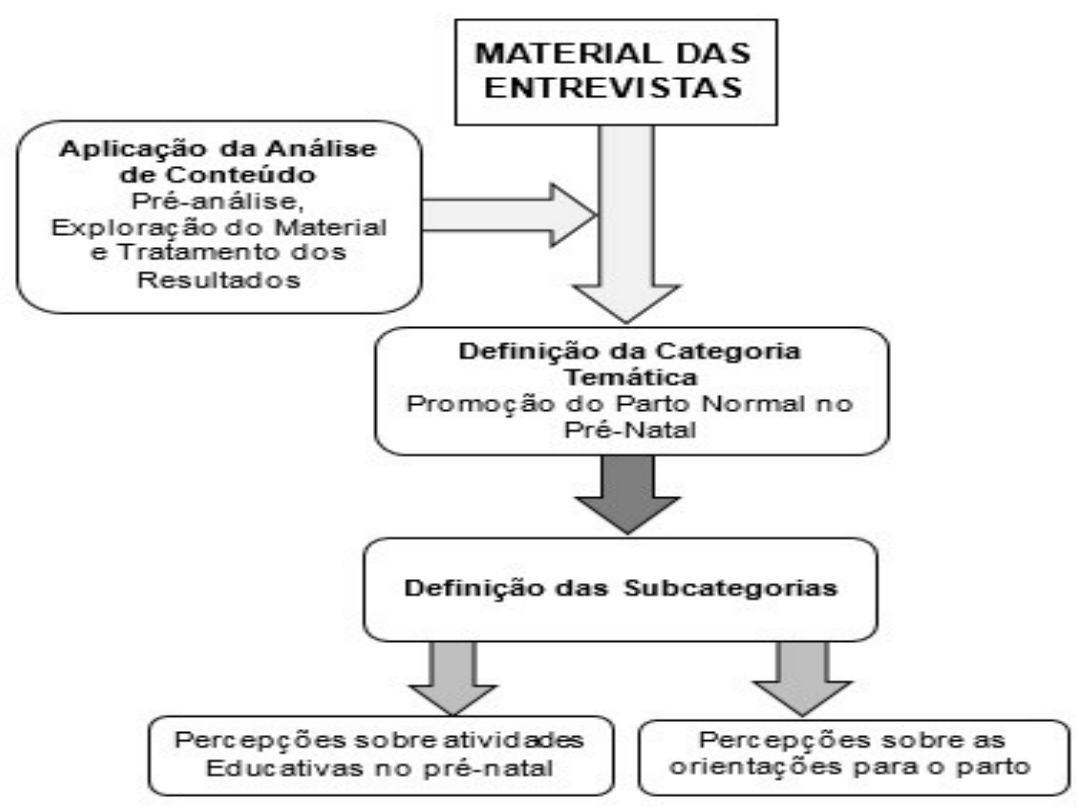

Figura 1: Plano de análise. Natal- RN, 2015.

\section{Perfil das gestantes}

Um perfil socioeconômico das participantes foi traçado. A tabela 1 apresenta os dados com mais detalhes.

\begin{tabular}{lll}
\hline \multicolumn{3}{c}{ Tabela 1: Distribuição do perfil socioeconômico das gestantes. } \\
Natal-RN, 2015.
\end{tabular}




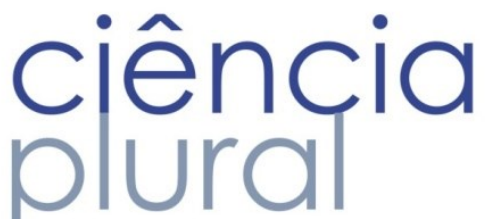

Em relação à idade, a faixa etária com maior porcentagem foi entre 24 e 29 anos (47,1\%), o que demonstra que a maior parte das participantes eram jovens e adultas. Quanto ao estado civil a maioria $(47,1 \%)$ era casada. Apenas $17,6 \%$ estavam com $\geq 14$ anos de estudo, o que infere que a maioria não possuía ensino superior. Já em relação ao trabalho, observou-se pouca diferença, umas vez que mais da metade (52,9\%) possuíam algum tipo de emprego. O perfil socioeconômico deste estudo vai ao encontro, em alguns pontos, de outros já realizados a nível nacional, que demonstra um público onde a maioria das entrevistadas era jovem e adulta; casada; com anos de estudos ou escolaridade que caracterizam minoria no ensino superior; mas contrapõem-se aos dados referentes às atividades laborais, onde a maioria caracteriza-se não possuir emprego ${ }^{11-14}$.

\section{CATEGORIA: PROMOÇÃO DO PARTO NORMAL NO PRÉ-NATAL}

\section{Subcategoria: Percepcões sobre atividades educativas no pré-natal}

O pré-natal acompanha o processo gravídico da mulher e o desenvolvimento fetal, além de possuir caráter de educação em saúde para promoção do parto normal. Neste estudo foi possível descrever a visão das gestantes em relação às atividades educativas quando questionadas: No pré-natal foram realizadas rodas de conversas, palestras ou grupos de gestantes em relação ao parto? A maioria delas referiu que houve poucas ou que não participou. As falas a seguir ilustram as respostas:

\footnotetext{
"Teve mais eu perdi...não me disseram nada, por certo esqueceram, e a agente de saúde não foi lá em casa, logo que eu trabalho o dia todinho". (Cerejeira).

“Já teve, mas eu não fui pra palestra”. (Amarílis).

"[...] até teve uma reunião, uma palestra, só que não pude vir que eu tava trabalhando". (Margarida).

"Teve uma palestra só que eu não pude vir". (Lírio).
}

Evidenciou-se que na assistência pré-natal das gestantes desta pesquisa foram desenvolvidas poucas atividades de educação em saúde. A falta de adesão às atividades educativas do pré-natal é clara, levantando hipóteses de insatisfação ou falta de vínculo da gestante à este serviço ou falta de busca ativa por parte dos profissionais. É necessário também, flexibilidade de dias e horários para as gestantes que trabalham, além do fortalecimento e a antecipação da divulgação dessas atividades, bem como considerar as necessidades apontadas pelas gestantes para o direcionamento dos temas e métodos utilizados nessas ocasiões. 


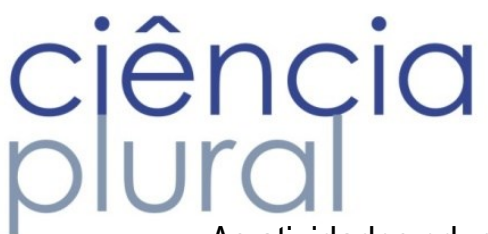

As atividades educativas, como palestras, grupo de gestantes ou rodas de conversa são importantes, pois favorecem a reflexão sobre um tema e possibilitam que cada indivíduo busque formas mais satisfatórias de lidar com as dificuldades comuns discutidas no grupo. A educação em saúde no pré-natal quando realizada com qualidade e comprometimento da equipe de saúde gera adesão das mulheres, isso porque elas atribuem grande importância às atividades de educação em saúde ${ }^{15}$. No entanto, foi evidenciada a falta dessa pratica educativa de forma que as mulheres por vezes chegam ao momento do parto despreparadas para vivenciá-lo. Vale salientar que as gestantes atribuem grande importância às atividades de educação em saúde ${ }^{16-17}$.

Quando questionadas em quais pontos o pré-natal poderia melhorar, os aspectos apontados foram diversos. No entanto, foram bastante frequentes as falas que destacaram a necessidade de melhor comunicação entre os profissionais da assistência e as gestantes. As falas a seguir demonstram:

"[...] identificar as coisas que vão acontecer e nos orientar, pra o momento tão esperado que é a chegada do bebê, muitos não têm aquela conversa, não tem aquele diálogo, só faz a consulta normal e pronto. Pesa, vê o tamanho, escuta coração, mas não tem aquele diálogo, de conversar, orientar, de preparar até a chegada do bebê". (Tulipa).

"Alguém da equipe que desse informações, eu acho que até tem, mas muitas vezes não são orientadas né, nem pra passar pra gente, nem pra gente perguntar a eles e eles terem uma resposta mais adequada". (Calêndula).

"Mais conversa né? Mais explicação". (Hibisco).

Assim, infere-se que o principal causador de falhas no processo de informação durante o pré-natal é a falta de diálogo entre o profissional de saúde e a gestante. E isso pode gerar sentimentos de ansiedade, medo ou insegurança. Esse resultado vai ao encontro de outros estudos, que também apontam a pouca ou mesmo ausência de diálogo como principal ponto de insatisfação, o que dificulta que as mulheres manifestem suas queixas, dúvidas e medos relacionados à gravidez ${ }^{18-19}$.

Estas falhas denotam a insuficiência da assistência pré-natal quanto ao papel esclarecedor e sensibilizador da educação em saúde, o que corrobora com a falta de conhecimento da mulher sobre a gestação, parto e puerpério. Vale ressaltar que o Ministério da Saúde preconiza que a relação interpessoal e a comunicação entre os profissionais da equipe de saúde e a gestante devem ser favorecidas com a inclusão de atividades educativas de grupo ${ }^{20}$. 


\section{Subcategoria: Percepções sobre as orientações para o parto normal}

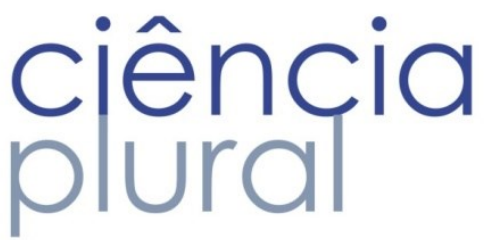

Considerando a necessidade do preparo para parturição e a importância das orientações para o parto que devem ser realizadas no pré-natal, foi questionado às gestantes se elas acreditam ser importante receber orientações sobre o parto normal durante o pré-natal e se elas receberam tais informações. As falas a seguir remetem ao questionamento:

"É importante, queria muito ter sido". (Cerejeira).

"É, principalmente "pra" mãe de primeira viajem." (Amarílis).

"Considero (importante), antes de ter é bem melhor né, porque aí já vai sabendo como é que é mais ou menos". (Íris).

"Sim, considero, apesar de não querer muito o parto normal, mas eu considero bastante importante né?". (Margarida).

"Sim, é bom à gente saber, por que a gente às vezes não sabe, mesmo que tenha experiência, mas a gente quer saber né". (Camélia).

"Sim, pra pessoa pelo menos já saber, ter noção do que vai acontecer, por que a pessoa vai ter um neném e não sabe nem como é que vai acontecer, como eles vão Ihe atender, como é que vai ser, mas não tem". (Hibisco).

As falas demonstram que as gestantes entendem a importância da preparação da gravidez para 0 parto, porém, os relatos apresentam que muitas ainda não haviam recebido qualquer tipo de informação ou orientação. Há falhas na preparação da mulher para o parto e isso ocorre no pré-natal, muitas vezes a gestante até necessita de apoio psicoemocional e não recebe ${ }^{21-22}$.

Estes dados apontam para a necessidade de implementação e intensificação de atividades educativas às gestantes, capazes de proporcioná-las conhecimento sobre seu corpo e das alterações que ocorrem durante o parto ${ }^{16}$. Certamente, as orientações para o parto normal propiciam à mulher o resgate da autoconfiança no seu corpo e na sua capacidade de parir, preparando-a para o enfrentamento da dor e encorajando-a a vivenciar a parturição com protagonismo.

Indagou-se quanto a melhor forma, considerada pelas entrevistadas, de educação em saúde no prénatal. Nesse sentido, os discursos foram frequentes apontando a palestra e o uso de aparelhos audiovisuais no auxílio das orientações, que contribuem para uma melhor demonstração do tema a ser abordado, facilitando o processo de assimilação e aprendizagem. As falas a seguir retratam isso:

"É, deveria ser na palestra, porque cada uma tinha uma opinião diferente [...]". (Cerejeira). 
"Mostrando, podia ser assim... em computador, [...] um vídeo, é! "Pra" gente poder ver, porque eu já vi [...] eu já vi parto normal na internet, só que eu não sei se é do mesmo jeito [...]". (Amarílis).

"Acho que em palestras, vinha todo mundo, eu acho que em palestras era melhor". (Gérbera).

"Mostrando, intensificando essas palestras que é muito importante e tipo na divulgação também, por que muitas mães acabam perdendo por não saber que tem, os dias entendeu? É por que muita gente não sabe quando tem". (Azaléia).

"Deveria ser palestras mesmo, por que muita coisa a gente vê às vezes na TV ou alguém fala e a gente não sabe se é verdade ou se é mentira, e eles falando é mais certo. Até por que a gente já vem pra isso né?". (Camélia).

"Palestras, vídeos, por que a gente vendo entra mais na nossa mente né. Eu acho que palestra resolve muito, por que tem muita mãe que às vezes não sabe nem por onde agir, mãe de primeira viagem, [...] a gente ainda se sente perdida, mas quando a gente vai pra uma palestra e o profissional lhe orienta é sempre bom, você já vai preparada". (Açucena).

É importante considerar a opinião das gestantes, público ao qual se destina a assistência pré-natal, e que deve ter sua percepção e opinião valorizada nesse processo. A melhor forma que consideram para a orientação do parto normal demonstra a valorização das atividades em grupo, com saberes compartilhados e da utilização de meios de tecnologia e recursos como facilitadores do aprendizado. Estudos apresentam o uso de rodas de conversas, sala de espera, uso de instrumentos audiovisuais e impressos como meios utilizados para educação em saúde e que se configuram formas de promoção do parto no pré-natal16, 23-24.

\section{Considerações Finais}

A necessidade de conhecer a percepção da gestante sobre a promoção do parto normal no pré-natal neste estudo torna-se válida, uma vez que é fundamental para o alcance de melhores resultados ao entender a visão das gestantes que vivenciam as práticas preconizadas pelo Ministério da Saúde e Organização Mundial da Saúde para a assistência de qualidade do cuidado pré-natal realizada pelas equipes multidisciplinares.

Foi possível se observar que ainda existem lacunas que permitem a falta de informação, gerando dúvidas e insatisfação nas gestantes. Assim, os resultados deste estudo apontam para a reorientação das ações de incentivo ao parto normal no pré-natal, e para a adoção de estratégias metodológicas claras e objetivas que subsidiem o trabalho dos profissionais, na atenção primária, para fazerem educação em saúde 


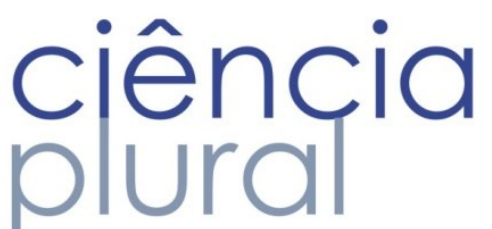

na assistência pré-natal. É preciso compreender as necessidades e dificuldades de acesso das gestantes e propiciá-las com incentivos capazes de fortalecer os vínculos com os profissionais e a atenção primária.

Recomenda-se, portanto, a realização de ações educativas e preparo para o parto normal em grupo, subsidiadas por um programa de atividades que considere as necessidades do grupo e que oferte a integração da equipe (para uma assistência integral à gestante), para que se alcance o fortalecimento do vínculo com os profissionais, a satisfação e adesão das gestantes. É importante proporcionar, também, instruções e orientações com ênfase no aconselhamento e apoio emocional, tão necessárias para a experiência do parto que a gestante vivenciará.

\section{Referências}

1. Leal MC, Pereira APE, Domingues RMSM, Filha Theme MM, Dias MAB, Pereira-Nakamura M, et al. Intervenções obstétricas durante o trabalho de parto e parto. Cad. Saúde Pública. 2014;30( Suppl 1):17-32.

2. Aquino EML. Para reinventar o parto e o nascimento no Brasil: de volta ao futuro. Cad. Saúde Pública. [online]. 2014; 30( Suppl 1):8-10.

3. Domingues RMSM, Dias AB, Pereira-Nakamura M, Torres JA, d'Orsi E, et al. Processo de decisão pelo tipo de parto no Brasil: da preferência inicial das mulheres à via de parto final. Cad. Saúde Pública. 2014;30(Suppl):101-116.

4. Organização Mundial da Saúde. Declaração da OMS sobre Taxas de Cesáreas. [Internet]; 2015 [citado em 2017 Set 20]. Disponível em:

http://apps.who.int/iris/bitstream/10665/161442/3/WHO_RHR_15.02_por.pdf

5. Oliveira RR, Melo EC, Novaes ES, Ferracioli PLRV, Mathias TAF. Fatores associados ao parto cesárea nos sistemas público e privado de atenção à saúde. Revista de Enfermagem da USP. 2016;50(5):734-741.

6. Nascimento RRP, Arantes SL, Souza EDC, Contrera L, Sales APA. Escolha do tipo de parto: fatores relatados por puérperas. Rev Gaúcha Enferm. 2015; 36(Esp):119-126.

7. Brasil. Ministério da Saúde. Secretaria de atenção à Saúde. Programa de humanização do parto, humanização do pré-natal e nascimento / Manual técnico. Ministério da Saúde. Brasília: Ministério da Saúde, 2002b.

8. Brasil. Ministério da Saúde. Universidade Estadual do Ceará. Humanização do parto e do nascimento. Ministério da Saúde. Brasília: Ministério da Saúde, 2014.

9. Brasil. Ministério da Saúde. Secretaria de Atenção à Saúde. Departamento de Atenção Básica. Atenção ao pré-natal de baixo risco. Ministério da Saúde. Brasília: 2012a.

10. Gomes R. A análise de dados em pesquisa qualitativa. In: Minayo MCS, Deslandes SF, Gomes R. Pesquisa Social: Teoria, método e criatividade. 30. ed. Petrópolis, RJ: Vozes; 2012. p. 67-80. 


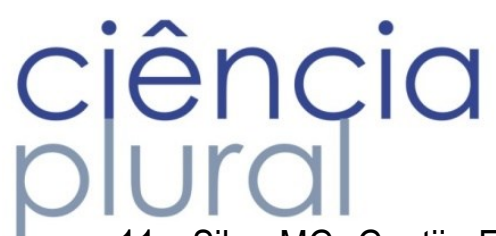

11. Silva MG, Gontijo EEL, Ferreira DS, Carvalho FS, Castro AM. O perfil epidemiológico de gestantes atendidas nas unidades básicas de saúde de Gurupi, Tocantins*. Universitas: Ciências da Saúde. 2015;13(2): 93-102.

12. Peixoto CR, Lima TM, Costa CC, Freitas LV, Oliveira AS, Damasceno AC. Perfil das gestantes atendidas no serviço de pré-natal das unidades básicas de saúde de fortaleza- CE. Rev. Min. Enferm. 2012;16(2):171-177.

13. Gomes VTS, Gomes RNS, Gomes MS, Lago EC, Caldas DRC, Santos CNC. Perfil nutricional e socioeconômico de gestantes assistidas em unidades básicas de saúde de Caxias/MA. R. Interd. 2015; $8(4): 127-135$.

14. Alves CN, Ressel LB, Sanfelice $C$, Bisognin P, Wilhelm LA, Zanini RR. Perfil de gestantes assistidas no pré-natal de enfermagem de uma unidade básica de saúde. J.res.: fundam. care. online. 2013;5(3):132-141.

15. Silva EAT. Gestação e preparo para o parto: programas de intervenção. O Mundo da Saúde. 2013;37( 2):208-215.

16. Souza VB, Roecker S, Marconi SS. Ações educativas durante a assistência pré-natal: percepção de gestantes atendidas na rede básica de Maringá-PR. Rev. Eletr. Enf. 2011;13(2):199-210.

17. Silva ALS; Nascimento ER, Coelho EAC, Nunes IM. Atividades educativas no pré-natal sob o olhar de mulheres grávidas. Revista Cubana de Enfermería. 2015;30(1).

18. Costa AP, Bustorff LACV, Cunha ARR, Soares CS, Araújo VS. Contribuições do pré-natal para o parto vaginal: percepção de puérperas. Rev Rene. 2011;12(3):548-554.

19. Guerreiro EM, Rodrigues AP, Silveira MAM, Lucena NBF. O cuidado pré-natal na atenção básica de saúde sob o olhar de gestantes e enfermeiros. Rev. Min. Enferm. 2012;16(3):315-323.

20. Brasil. Ministério da Saúde. Secretaria de Atenção à Saúde. Departamento de Ações Programáticas Estratégicas. Área Técnica de Saúde da Mulher. Pré-natal e Puerpério: atenção qualificada e humanizada. Manual técnico / Ministério da Saúde. Brasília: Ministério da Saúde, 2006.

21. Brito CA, Silva ASS, Cruz RSBLC, Pinto SL. Percepções de puérperas sobre a preparação para o parto no pré-natal. Rev Rene. 2015; 16(4):470-8.

22. Tostes NA, Seidl EMF. Expectativas de gestantes sobre o parto e suas percepções acerca da preparação para o parto. Temas psicol. 2016; 24(2):681-693.

23. Santos DS, Andrade ALA, Lima ABSS, Silva YN. Sala de espera para gestantes: uma estratégia de educação em saúde. Revista Brasileira de Educação Médica. 2012;36 (1 Suppl 2): 62-67.

24. Fagundes $D Q$, Oliveira $A E$. Educação em saúde no pré-natal a partir do referencial teórico de Paulo Freire. Trab. Educ. Saúde. 2017;15 (1): 223-243. 